\title{
OPE of the energy-momentum tensor correlator and the gluon condensate operator in massless QCD to three-loop order
}

\section{M.F. Zoller}

Institut für Theoretische Teilchenphysik, Karlsruhe Institute of Technology (KIT),

D-76128 Karlsruhe, Germany

E-mail: max.zoller@kit.edu

ABSTRACT: The correlator of two gluonic operators plays an important role for example in transport properties of a Quark Gluon Plasma (QGP) or in sum rules for glueballs.

In [1] an operator product expansion (OPE) at zero temperature was performed for the correlators of two scalar operators $O_{1}=-\frac{1}{4} G^{\mu \nu} G_{\mu \nu}$ and two QCD energy-momentum tensors $T^{\mu \nu}$. There we presented analytical two-loop results for the Wilson coefficient $C_{1}$ in front of the gluon condensate operator $O_{1}$. In this paper these results are extended to three-loop order.

The three-loop Wilson coefficient $C_{0}$ in front of the unity operator $O_{0}=\mathbb{1}$ was already presented in [1] for the $T^{\mu \nu}$-correlator. For the $O_{1}$-correlator the coefficient $C_{0}$ is known to four loop order from [2]. For the correlator of two pseudoscalar operators $\tilde{O}_{1}=\varepsilon_{\mu \nu \rho \sigma} G^{\mu \nu} G^{\rho \sigma}$ both coefficients $C_{0}$ and $C_{1}$ were computed in [3] to three-loop order. At zero temperature $C_{0}$ and $C_{1}$ are the leading Wilson coefficients in massless QCD.

KeYwords: QCD, Sum Rules, Quark-Gluon Plasma

ARXiv EPRINT: 1407.6921 


\section{Contents}

1 Introduction and definitions 1

2 Calculation and results 4

$\begin{array}{lll}3 & \text { Numerics } & 11\end{array}$

4 Conclusions $\quad 13$

\section{Introduction and definitions}

Correlators of two local operators $O(x)$ are important objects in quantum field theory. In momentum space they are defined as

$$
i \int \mathrm{d}^{4} x e^{i q x} T\{[O](x)[O](0)\},
$$

where $[O]$ ist defined to be a renormalized version of the operator $O$, i.e. matrix elements of $[O]$ are finite. ${ }^{1}$ For sum rules we are usually interested in the vacuum expectation value (VEV) of the correlator

$$
\left.\Pi\left(Q^{2}\right)=i \int \mathrm{d}^{4} x e^{i q x}\langle 0| T\{[O](x)[O](0)]\right\}|0\rangle
$$

with large $Q^{2}:=-q^{2}>0$, i.e. in the Euclidean region of momentum space. The function $\Pi\left(Q^{2}\right)$ is connected to the spectral density $\operatorname{Im} \Pi(s)$ in the region of physical momenta through a dispersion relation (see e.g. [4]).

The leading contribution to $\Pi\left(Q^{2}\right)$ can be computed perturbatively and is exactly the first Wilson coefficient in front of the unity operator $O_{0}=\mathbb{1}$. In order to include nonperturbative effects as well the correlator (1.1) is expanded in a series of local operators with Wilson coefficients containing the dependence on $q$ in momentum space or $x$ in $\mathrm{x}$ space [5]. This operator product expansion (OPE) has the form

$$
\begin{aligned}
i \int \mathrm{d}^{4} x e^{i q x} T\{[O](x)[O](0)\} & =\sum_{i}\left(Q^{2}\right)^{\frac{2 \operatorname{dim}(O)-\operatorname{dim}\left(O_{i}\right)-4}{2}} C_{i}^{\mathrm{B}}(q) O_{i}^{\mathrm{B}} \\
& =\sum_{i}\left(Q^{2}\right)^{\frac{2 \operatorname{dim}(O)-\operatorname{dim}\left(O_{i}\right)-4}{2}} C_{i}(q)\left[O_{i}\right]
\end{aligned}
$$

\footnotetext{
${ }^{1}$ By a local operator $O$ we mean a combination of fields at the same space-time point. The bare operator $O^{\mathrm{B}}$ is the same combination but with bare fields and in the simplest case $[O]=Z^{O} O^{\mathrm{B}}$ is the renormalized operator with a renormalization constant $Z^{O}$. In some cases a set of operators mixes under renormalization giving $\left[O_{i}\right]=Z_{i j}^{O} O_{j}^{\mathrm{B}}$, where all $\left[O_{i}\right]$ are finite if inserted into a Greens function. If more than one operator is inserted into a Greens function additional divergences may appear if these operators are taken to be at the same space-time point. Such contributions are called contact terms.
} 
where the index B marks bare quantities. The factor $\left(Q^{2}\right)^{\frac{2 \operatorname{dim}(O)-\operatorname{dim}\left(O_{i}\right)-4}{2}}$ is constructed from the mass dimensions of the operators in order to make $C_{i}(q)$ dimensionless.

The perturbative contribution is separated from the non-perturbative condensates in an operator product expansion (OPE) and hence resides in the Wilson coefficients in front of local operators. These Wilson coefficients are calculated perturbatively using the method of projectors $[6,7]$ and contain the perturbative contribution to the correlator in question. If we insert expansion (1.4) into (1.2) we are left with the task of determining the VEVs of the local operators $\left[O_{i}\right]$, the so-called condensates [8], which contain the non-perturbative part. These need to be derived from low energy theorems or be calculated on the lattice.

Three gluonic operators with the quantum numbers $J^{\mathrm{PC}}=0^{++}, 0^{-+}$and $2^{++}$are usually considered: ${ }^{2}$

$$
\begin{aligned}
O_{1}^{\mathrm{B}}(x) & =-\frac{1}{4} G^{\mathrm{B} a \mu \nu} G_{\mu \nu}^{\mathrm{B} a}(x) & (\text { scalar }), \\
\tilde{O}_{1}^{\mathrm{B}}(x) & =\varepsilon_{\mu \nu \rho \sigma} G^{\mathrm{B} a \mu \nu} G^{\mathrm{B} a \rho \sigma}(x) & \text { (pseudoscalar) } \\
O_{T}^{\mu \nu}(x) & =T^{\mu \nu}(x) & \text { (tensor) }
\end{aligned}
$$

with the bare gluon field strength tensor

$$
G_{\mu \nu}^{a \mathrm{~B}}=\partial_{\mu} A_{\nu}^{a \mathrm{~B}}-\partial_{\nu} A_{\mu}^{a \mathrm{~B}}+g_{s}^{\mathrm{B}} f^{a b c} A_{\mu}^{\mathrm{B} b} A_{\nu}^{\mathrm{B} c},
$$

where $f^{a b c}$ are the structure constants and $T^{a}$ the generators of the $\mathrm{SU}\left(N_{c}\right)$ gauge group. As described in [1] for $T^{\mu \nu}$ we use the gauge invariant and symmetric energy-momentum tensor of (massless) QCD:

$$
\begin{aligned}
\left.T_{\mu \nu}\right|_{\text {ginv }}= & -G_{\mu \rho}^{\mathrm{B} a} G_{\nu}^{\mathrm{B} a \rho}+\frac{i}{4} \bar{\psi}^{\mathrm{B}}\left(\overleftrightarrow{\partial_{\mu}} \gamma_{\nu}+\overleftrightarrow{\partial_{\nu}} \gamma_{\mu}\right) \psi^{\mathrm{B}}+\frac{1}{2} g_{s}^{\mathrm{B}} \bar{\psi}^{\mathrm{B}}\left(A_{\mu}^{\mathrm{B} a} T^{a} \gamma_{\nu}+A_{\nu}^{\mathrm{B} a} T^{a} \gamma_{\mu}\right) \psi^{\mathrm{B}} \\
& -g_{\mu \nu}\left\{-\frac{1}{4} G_{\rho \sigma}^{\mathrm{B} a} G^{\mathrm{B} a \rho \sigma}+\frac{i}{2} \bar{\psi}^{\mathrm{B}} \overleftrightarrow{\not} \psi^{\mathrm{B}}+g_{s} \bar{\psi}^{\mathrm{B}} \dot{A}^{\mathrm{B} a} T^{a} \psi^{\mathrm{B}}\right\} .
\end{aligned}
$$

In [13] it was argued that if we are only interested in matrix elements of only gauge invariant operators it is not necessary to consider the ghost terms appearing in the full energy-momentum tensor of QCD. It was also proven that the energy-momentum tensor of QCD is a finite operator without further renormalization.

The operator $O_{1}$ and the Wilson coefficients $C_{1}$, however, have to be renormalized in the following way:

$$
\begin{aligned}
{\left[O_{1}\right] } & =Z_{G} O_{1}^{\mathrm{B}}=-\frac{Z_{G}}{4} G^{\mathrm{B} a \mu \nu} G_{\mu \nu}^{\mathrm{B} a} \\
C_{1} & =\frac{1}{Z_{G}} C_{1}^{\mathrm{B}} .
\end{aligned}
$$

\footnotetext{
${ }^{2}$ For details on the sum rule approach to glueballs with the same quantum numbers see e.g. [4]. An OPE at one-loop level has been performed for the scalar [9] and pseudoscalar [10] correlator. Recent discussions on glueballs using an OPE of these correlators can be found in $[11,12]$.
} 
The renormalization constant

$$
Z_{G}=1+\alpha_{s} \frac{\partial}{\partial \alpha_{s}} \ln Z_{\alpha_{s}}=\left(1-\frac{\beta\left(\alpha_{s}\right)}{\varepsilon}\right)^{-1}
$$

was derived in $[14,15]$ from the renormalization constant $Z_{\alpha_{s}}$ for $\alpha_{s}$. At first order in $\alpha_{s}$ we find $Z_{G}=Z_{\alpha_{s}}$, which is not true in higher orders however. We take the definition

$$
\beta\left(\alpha_{s}\right)=\mu^{2} \frac{\mathrm{d}}{\mathrm{d} \mu^{2}} \ln \alpha_{s}=-\sum_{i \geq 0} \beta_{i}\left(\frac{\alpha_{s}}{\pi}\right)^{i+1}
$$

for the $\beta$-function of QCD, which is available at four-loop level [16, 17]. For the renormalization of $\tilde{O}_{1}^{\mathrm{B}}$, which mixes with a pseudoscalar fermionic operator under renormalization, and its OPE we refer to [3, 18].

The correlators of $O_{1}$ and $O_{T}^{\mu \nu}$ have been discussed in [1], where $C_{1}$ has been presented at two-loop level. The results of this work are derived within the same theoretical and methodical framework, which is why we can refer to this work for most technical details. $C_{0}$ is also known to three-loop level for the $T^{\mu \nu}$-correlator [1] and at two-, three- and fourloop level for the $O_{1}$-correlator from [19], [20] and [2] respectively. Three-loop results for $C_{0}$ and $C_{1}$ for the correlator of two operators $\tilde{O}_{1}$ have been derived in [3].

The VEV of the energy-momentum tensor correlator

$$
\begin{aligned}
& T^{\mu \nu ; \rho \sigma}(q):=\left\langle 0\left|\hat{T}^{\mu \nu ; \rho \sigma}(q)\right| 0\right\rangle, \\
& \hat{T}^{\mu \nu ; \rho \sigma}(q):=i \int \mathrm{d}^{4} x e^{i q x} T\left\{T^{\mu \nu}(x) T^{\rho \sigma}(0)\right\}
\end{aligned}
$$

is an important quantitiy in calculations of transport properties of a Quark Gluon Plasma (QGP), such as the shear viscosity of the plasma (see e.g. [21, 22]) and spectral functions for some tensor channels in the QGP [23].

The correlator (1.15) is linked to the $O_{1}$-correlator

$$
Q^{4} \Pi^{\mathrm{GG}}\left(q^{2}\right):=i \int \mathrm{d}^{4} x e^{i q x}\left\langle 0\left|T\left[O_{1}(x) O_{1}(0)\right]\right| 0\right\rangle
$$

through the trace anomaly $[13,24]$

$$
T_{\mu}^{\mu}=\frac{\beta\left(a_{s}\right)}{2}\left[G_{\rho \sigma}^{a} G^{a \rho \sigma}\right]=-2 \beta\left(a_{s}\right)\left[O_{1}\right],
$$

which leads to

$$
g_{\mu \nu} g_{\rho \sigma} T^{\mu \nu ; \rho \sigma}(q)=4 \beta^{2}\left(\alpha_{s}\right) Q^{4} \Pi^{\mathrm{GG}}\left(q^{2}\right)+\text { contact terms. }
$$

Both correlators $\Pi^{\mathrm{GG}}$ and $T^{\mu \nu ; \rho \sigma}(q)$ have been studied in hot Yang-Mills theory in many works, see e.g. [25-29] and references therein.

At zero temperature (1.15) has the asymptotic behaviour

$$
\hat{T}^{\mu \nu ; \rho \sigma}(q) \underset{q^{2} \rightarrow-\infty}{\rightleftharpoons} C_{0}^{\mu \nu ; \rho \sigma}(q) \mathbb{1}+C_{1}^{\mu \nu ; \rho \sigma}(q)\left[O_{1}\right]+\ldots
$$


where the tensor structure of the correlator resides in the Wilson coefficients if we are ultimately only interested in the VEV of the correlator.

Local tensor operators can always be decomposed in a trace part and a traceless part, i.e. for two Lorentz indices

$$
O^{\mu \nu}=\underbrace{O^{\mu \nu}-\frac{1}{D} g^{\mu \nu} O_{\rho}^{\rho}}_{\text {traceless part }}+\underbrace{\frac{1}{D} g^{\mu \nu} O_{\rho}^{\rho}}_{\text {trace part }}
$$

where $D$ is the dimension of the space time. The VEV of the traceless part vanishes due to the Lorentz invariance of the vacuum and only a local scalar operator $O_{\rho}^{\rho}$ survives.

The OPE of the correlator (1.16) reads

$$
Q^{4} \Pi^{\mathrm{GG}}\left(q^{2}\right) \underset{q^{2} \rightarrow-\infty}{\rightleftharpoons} C_{0}^{\mathrm{GG}} Q^{4}+C_{1}^{\mathrm{GG}}\left\langle 0\left|\left[O_{1}\right]\right| 0\right\rangle .
$$

\section{Calculation and results}

As discussed in [1] there are five independent tensor structures for (1.19) allowed by the symmetries $\mu \longleftrightarrow \nu, \rho \longleftrightarrow \sigma$ and $(\mu \nu) \longleftrightarrow(\rho \sigma)$ of (1.19). These are

$$
\begin{aligned}
& t_{1}^{\mu \nu ; \rho \sigma}(q)=q^{\mu} q^{\nu} q^{\rho} q^{\sigma}, \\
& t_{2}^{\mu \nu ; \rho \sigma}(q)=q^{2}\left(q^{\mu} q^{\nu} g^{\rho \sigma}+q^{\rho} q^{\sigma} g^{\mu \nu}\right), \\
& t_{3}^{\mu \nu ; \rho \sigma}(q)=q^{2}\left(q^{\mu} q^{\rho} g^{\nu \sigma}+q^{\mu} q^{\sigma} g^{\nu \rho}+q^{\nu} q^{\rho} g^{\mu \sigma}+q^{\nu} q^{\sigma} g^{\mu \rho}\right), \\
& t_{4}^{\mu \nu ; \rho \sigma}(q)=\left(q^{2}\right)^{2} g^{\mu \nu} g^{\rho \sigma}, \\
& t_{5}^{\mu \nu ; \rho \sigma}(q)=\left(q^{2}\right)^{2}\left(g^{\mu \rho} g^{\nu \sigma}+g^{\mu \sigma} g^{\nu \rho}\right) .
\end{aligned}
$$

Due to the fact that the energy-momentum tensor is conserved except for contact terms, i.e.

$$
q_{\mu} T^{\mu \nu ; \rho \sigma}(q)=\text { local contact terms, }
$$

and due to the irrelevance of these contact terms for physical applications we can reduce (2.1) to only two independent tensor structures, which have already been suggested in [30], after contact term subtraction: :

$$
\begin{aligned}
& t_{S}^{\mu \nu ; \rho \sigma}(q)=\eta^{\mu \nu} \eta^{\rho \sigma} \\
& t_{T}^{\mu \nu ; \rho \sigma}(q)=\eta^{\mu \rho} \eta^{\nu \sigma}+\eta^{\mu \sigma} \eta^{\nu \rho}-\frac{2}{D-1} \eta^{\mu \nu} \eta^{\rho \sigma}
\end{aligned}
$$

$$
\text { with } \eta^{\mu \nu}(q)=q^{2} g^{\mu \nu}-q^{\mu} q^{\nu} .
$$

The structure $t_{T}^{\mu \nu ; \rho \sigma}(q)$ is traceless and orthogonal to $t_{S}^{\mu \nu ; \rho \sigma}(q)$. Hence the latter corresponds to the part coming from the traces of the energy-momentum tensors. The Wilson coefficient in front of the local operator $\left[O_{1}\right]$ has the form

$$
\begin{aligned}
C_{1}^{\mu \nu ; \rho \sigma}(q) & =\sum_{r=1,5} t_{r}^{\mu \nu ; \rho \sigma}(q) \frac{1}{\left(Q^{2}\right)^{2}} C_{i}^{(r)}\left(Q^{2}\right) \\
& =\sum_{r=T, S} t_{r}^{\mu \nu ; \rho \sigma}(q) \frac{1}{\left(Q^{2}\right)^{2}} C_{i}^{(r)}\left(Q^{2}\right) \text { (+ contact terms) }
\end{aligned}
$$


where the contact terms have to be $\propto t_{4}^{\mu \nu ; \rho \sigma}(q)$ or $\propto t_{5}^{\mu \nu ; \rho \sigma}(q)$ as $t_{r}^{\mu \nu ; \rho \sigma}(q) \frac{1}{\left(Q^{2}\right)^{2}}$ is not local for $r \in\{1,2,3\}$. This was checked explicitly in our three-loop result.

Just like in [1] (see this paper for more details) the method of projectors [6, 7] was used in order to compute the coefficient $C_{1}^{\mu \nu ; \rho \sigma}(q)$. We apply the same projector to both sides of (1.3):

$$
\mathbf{P}\left\{i \int \mathrm{d}^{4} x e^{i q x} T\{[O](x)[O](0)\}\right\}=\sum_{i} C_{i}^{\mathrm{B}}(q) \mathbf{P}\left\{O_{i}^{\mathrm{B}}\right\} \stackrel{!}{=} C_{1}^{\mathrm{B}}(q) .
$$

The projector $\mathbf{P}$ is constructed in such a way that it maps every operator on the r.h.s. of (1.3) to zero except for $O_{1}^{\mathrm{B}}$, which is mapped to 1 and hence gives us the bare Wilson coefficient $C_{1}^{\mathrm{B}}$ on the l.h.s.. For the $T^{\mu \nu}$-correlator (1.15) this is done after contracting the free Lorentz indices with a tensor $\tilde{t}_{\mu \nu ; \rho \sigma}^{(r)}(q)$ composed of the momentum $q$ and the metric $g^{\mu \nu}$ in order to get the scalar pieces in $(2.4):^{3}$

$$
\mathbf{P}\left\{\tilde{t}_{\mu \nu ; \rho \sigma}^{(r)}(q) T^{\mu \nu ; \rho \sigma}(q)\right\}=\sum_{i} C_{i}^{\mathrm{B},(r)}\left(Q^{2}\right) \mathbf{P}\left\{O_{i}^{\mathrm{B}}\right\} .
$$

We use the following projector: ${ }^{4}$

$$
C_{1}^{\mathrm{B}}(q)=\frac{\delta^{a b}}{n_{g}} \frac{g^{\mu_{1} \mu_{2}}}{(D-1)} \frac{1}{D} \frac{\partial}{\partial k_{1}} \cdot \frac{\partial}{\partial k_{2}}\left[\begin{array}{c}
\mu_{g_{B}} \\
\mu_{1}
\end{array}\right] \mu_{g_{B}}
$$

where the blue circle represents the sum of all bare Feynman diagrams which become 1PI after formal gluing (depicted as a dotted line in (2.7)) of the two external lines representing the operators on the l.h.s. of the OPE. These external legs carry the large Euclidean momentum q.

In order to produce all possible Feynman diagrams we have used the program QGRAF [32]. These propagator-like diagrams were computed with the FORM [33, 34] package MINCER [35] after projecting them to scalar pieces. For the colour factors of the diagrams the FORM package COLOR [36] was used.

We now give the three-loop results for the Wilson coefficient $C_{1}$ of the correlators (1.15) and (1.16) in the $\overline{\mathrm{MS}}$-scheme. In the following the abbreviations $a_{s}=\frac{\alpha_{s}}{\pi}=\frac{g_{s}^{2}}{4 \pi^{2}}$ and $l_{\mu q}=\ln \left(\frac{\mu^{2}}{Q^{2}}\right)$ are used, where $\mu$ is the $\overline{\mathrm{MS}}$ renormalization scale. The number of active quark flavours is denoted by $n_{f}$. Furthermore, $C_{F}$ and $C_{A}$ are the quadratic Casimir operators of the quark and the adjoint representation of the gauge group, $d_{R}$ is the dimension of the quark representation, $n_{g}$ is the number of gluons (dimension of the adjoint representation), $T_{F}$ is defined through the relation $\operatorname{Tr}\left(T^{a} T^{b}\right)=T_{F} \delta^{a b}$ for the trace of two group

\footnotetext{
${ }^{3}$ The $\tilde{t}_{\mu \nu ; \rho \sigma}^{(r)}$ can be constructed as linear combinations of the $t_{r}^{\mu \nu ; \rho \sigma}(q)$ in $(2.1)$.

${ }^{4}$ The Feynman diagram has been drawn with the Latex package Axodraw [31].
} 
generators. $^{5}$

$$
\begin{aligned}
C_{1}^{(S)}=a_{s} & \left\{\frac{22 C_{A}}{27}-\frac{8 n_{f} T_{F}}{27}\right\} \\
+ & a_{s}^{2}\left\{\frac{83 C_{A}^{2}}{324}-\frac{8 C_{A} n_{f} T_{F}}{81}-\frac{2 C_{F} n_{f} T_{F}}{9}-\frac{4 n_{f}^{2} T_{F}^{2}}{81}\right\} \\
+ & a_{s}^{3}\left\{-\frac{466 C_{A}^{3}}{729}+\frac{1309 C_{A}^{2} n_{f} T_{F}}{1944}-\frac{7}{648} C_{A} C_{F} n_{f} T_{F}-\frac{313}{972} C_{A} n_{f}^{2} T_{F}^{2}\right. \\
& +\frac{1}{36} C_{F}^{2} n_{f} T_{F}-\frac{7}{162} C_{F} n_{f}^{2} T_{F}^{2}+\frac{20 n_{f}^{3} T_{F}^{3}}{729} \\
& \left.+l_{\mu q}\left(-\frac{1331 C_{A}^{3}}{3888}+\frac{121}{324} C_{A}^{2} n_{f} T_{F}-\frac{11}{81} C_{A} n_{f}^{2} T_{F}^{2}+\frac{4 n_{f}^{3} T_{F}^{3}}{243}\right)\right\} \\
C_{1}^{(T)}= & \left\{-\frac{5 C_{A}}{18}-\frac{5 n_{f} T_{F}}{72}\right\} \\
+ & a_{s}^{2}\left\{-\frac{83 C_{A}^{2}}{432}+\frac{41 C_{A} n_{f} T_{F}}{432}+\frac{43 C_{F} n_{f} T_{F}}{96}-\frac{n_{f}^{2} T_{F}^{2}}{216}\right\} \\
+ & a_{s}^{3}\left\{-\frac{3 C_{A}^{3} \zeta_{3}}{8}+\frac{103 C_{A}^{3}}{15552}-\frac{27}{80} C_{A}^{2} n_{f} T_{F} \zeta_{3}+\frac{72239 C_{A}^{2} n_{f} T_{F}}{103680}\right. \\
& +\frac{3}{8} C_{A} C_{F} n_{f} T_{F} \zeta_{3}+\frac{923 C_{A} C_{F} n_{f} T_{F}}{1728}-\frac{3}{40} C_{A} n_{f}^{2} T_{F}^{2} \zeta_{3}-\frac{217 C_{A} n_{f}^{2} T_{F}^{2}}{1620} \\
& -\frac{241}{768} C_{F}^{2} n_{f} T_{F}-\frac{21}{40} C_{F} n_{f}^{2} T_{F}^{2} \zeta_{3}+\frac{929 C_{F} n_{f}^{2} T_{F}^{2}}{17280}+\frac{5 n_{f}^{3} T_{F}^{3}}{1944} \\
& +l_{\mu q}\left(\frac{107 C_{A}^{3}}{5184}+\frac{73}{864} C_{A}^{2} n_{f} T_{F}+\frac{131}{384} C_{A} C_{F} n_{f} T_{F}-\frac{7}{108} C_{A} n_{f}^{2} T_{F}^{2}\right. \\
& \left.\left.-\frac{1}{6} C_{F} n_{f}^{2} T_{F}^{2}+\frac{n_{f}^{3} T_{F}^{3}}{648}\right)\right\}
\end{aligned}
$$

In [1] it was shown that up to two-loop level the coefficient $C_{1}^{(S)}$, which corresponds to the trace of the two energy-momentum tensors in the correlator (1.15), can be written in the form

$$
C_{1}^{(S)}=-\frac{8}{9} \beta\left(a_{s}\right)\left(1+\frac{\beta\left(a_{s}\right)}{2}\right)+\mathcal{O}\left(\alpha_{s}^{3}\right),
$$

where the first factor $\beta\left(a_{s}\right)$ is due to the trace anomaly (1.17). It is interesting to check whether we can find a similar structure in terms of the $\beta$-function at three-loop level. However, we do not find such an elegant representation at the next loop order. The closest we get is

$$
\begin{aligned}
C_{1}^{(S)}= & -\frac{8}{9} \beta\left(a_{s}\right)\left[1+\frac{\beta\left(a_{s}\right)}{2}-\left(\frac{5}{3}+l_{\mu q}\right) \frac{\beta\left(a_{s}\right)^{2}}{2}\right. \\
& \left.+a_{s}^{2}\left(-\frac{5 C_{A}^{2}}{18}+\frac{13 C_{A} n_{f} T_{F}}{72}+\frac{C_{F} n_{f} T_{F}}{8}\right)\right]+\mathcal{O}\left(\alpha_{s}^{4}\right) .
\end{aligned}
$$

\footnotetext{
${ }^{5}$ For an $\mathrm{SU}(N)$ gauge group these are $d_{R}=N, C_{A}=2 T_{F} N$ and $C_{F}=T_{F}\left(N-\frac{1}{N}\right)$. For QCD $(\mathrm{SU}(3))$ this means $C_{F}=4 / 3, C_{A}=3, T_{F}=1 / 2$ and $d_{R}=3$.
} 
From the renormalization group invariance (RGI) of the energy-momentum tensor and (1.17) follows that RGI invariant Wilson coefficients for RGI operators on the r.h.s. of the OPE (1.4) can be constructed as already explained in [1]. The scale invariant version of the operator $O_{1}$ is defined by

$$
O_{1}^{\mathrm{RGI}}:=\hat{\beta}\left(a_{s}\right)\left[O_{1}\right], \quad \hat{\beta}\left(a_{s}\right)=\frac{-\beta\left(a_{s}\right)}{\beta_{0}}=a_{s}\left(1+\sum_{i \geq 1} \frac{\beta_{i}}{\beta_{0}} a_{s}^{i}\right) .
$$

From this and the scale invariance of the correlator (1.15) RGI Wilson coefficients can be defined as

$$
\begin{aligned}
C_{1, \mathrm{RGI}}^{(S)} & :=C_{1}^{(S)} / \hat{\beta}\left(a_{s}\right), \\
C_{1, \mathrm{RGI}}^{(T)} & :=C_{1}^{(T)} / \hat{\beta}\left(a_{s}\right),
\end{aligned}
$$

such that

$$
C_{1, \mathrm{RGI}}^{(S, T)} O_{1}^{\mathrm{RGI}}=C_{1}^{(S, T)}\left[O_{1}\right] .
$$

We find

$$
\begin{aligned}
C_{1, \mathrm{RGI}}^{(S)}= & \frac{22 C_{A}}{27}-\frac{8 n_{f} T_{F}}{27} \\
+ & a_{s}\left\{-\frac{121 C_{A}^{2}}{324}+\frac{22 C_{A} n_{f} T_{F}}{81}-\frac{4 n_{f}^{2} T_{F}^{2}}{81}\right\} \\
+ & a_{s}^{2}\left\{-\frac{12661 C_{A}^{3}}{11664}+\frac{365}{324} C_{A}^{2} n_{f} T_{F}+\frac{11}{54} C_{A} C_{F} n_{f} T_{F}\right. \\
& -\frac{83}{243} C_{A} n_{f}^{2} T_{F}^{2}-\frac{2}{27} C_{F} n_{f}^{2} T_{F}^{2}+\frac{20 n_{f}^{3} T_{F}^{3}}{729} \\
& \left.+l_{\mu q}\left(-\frac{1331 C_{A}^{3}}{3888}+\frac{121}{324} C_{A}^{2} n_{f} T_{F}-\frac{11}{81} C_{A} n_{f}^{2} T_{F}^{2}+\frac{4 n_{f}^{3} T_{F}^{3}}{243}\right)\right\}
\end{aligned}
$$

and

$$
\begin{aligned}
C_{1, \mathrm{RGI}}^{(T)}=- & \frac{5 C_{A}}{18}-\frac{5 n_{f} T_{F}}{72} \\
+ & a_{s}\left\{\frac { 1 } { ( 1 1 C _ { A } - 4 n _ { f } T _ { F } ) } \left[\frac{107 C_{A}^{3}}{432}+\frac{73 C_{A}^{2} n_{f} T_{F}}{72}-2 C_{F} n_{f}^{2} T_{F}^{2}\right.\right. \\
& \left.\left.+\frac{131 C_{A} C_{F} n_{f} T_{F}}{32}+\frac{n_{f}^{3} T_{F}^{3}}{54}-\frac{7 C_{A} n_{f}^{2} T_{F}^{2}}{9}\right]\right\} \\
+ & a_{s}^{2}\left\{-\frac{3}{40} C_{A} n_{f}^{2} T_{F}^{2} \zeta_{3}-\frac{217 C_{A} n_{f}^{2} T_{F}^{2}}{1620}-\frac{241}{768} C_{F}^{2} n_{f} T_{F}-\frac{21}{40} C_{F} n_{f}^{2} T_{F}^{2} \zeta_{3}\right. \\
& +\frac{929 C_{F} n_{f}^{2} T_{F}^{2}}{17280}+\frac{5 n_{f}^{3} T_{F}^{3}}{1944}-\frac{3 C_{A}^{3} \zeta_{3}}{8}+\frac{103 C_{A}^{3}}{15552}-\frac{27}{80} C_{A}^{2} n_{f} T_{F} \zeta_{3} \\
& +\frac{72239 C_{A}^{2} n_{f} T_{F}}{103680}+\frac{3}{8} C_{A} C_{F} n_{f} T_{F} \zeta_{3}+\frac{923 C_{A} C_{F} n_{f} T_{F}}{1728} \\
& +\frac{1}{\left(11 C_{A}-4 n_{f} T_{F}\right)}\left[+\frac{1411 C_{A}^{4}}{864}-\frac{509 C_{A}^{3} n_{f} T_{F}}{288}-\frac{2525 C_{A}^{2} C_{F} n_{f} T_{F}}{576}\right.
\end{aligned}
$$




$$
\begin{aligned}
& \left.+\frac{37 C_{A}^{2} n_{f}^{2} T_{F}^{2}}{72}+\frac{43 C_{F}^{2} n_{f}^{2} T_{F}^{2}}{32}-\frac{C_{F} n_{f}^{3} T_{F}^{3}}{72}+\frac{727 C_{A} C_{F} n_{f}^{2} T_{F}^{2}}{288}-\frac{5 C_{A} n_{f}^{3} T_{F}^{3}}{216}\right] \\
& +\frac{1}{\left(11 C_{A}-4 n_{f} T_{F}\right)^{2}}\left[+\frac{53095 C_{A}^{5}}{5184}-\frac{308465 C_{A}^{4} n_{f} T_{F}}{20736}+\frac{965 C_{A}^{3} C_{F} n_{f} T_{F}}{864}\right. \\
& +\frac{10255 C_{A}^{3} n_{f}^{2} T_{F}^{2}}{3456}+\frac{55 C_{A}^{2} C_{F}^{2} n_{f} T_{F}}{48}-\frac{65 C_{A}^{2} C_{F} n_{f}^{2} T_{F}^{2}}{128}+\frac{3175 C_{A}^{2} n_{f}^{3} T_{F}^{3}}{5184} \\
& \left.-\frac{35 C_{F}^{2} n_{f}^{3} T_{F}^{3}}{48}-\frac{505 C_{A} C_{F}^{2} n_{f}^{2} T_{F}^{2}}{192}-\frac{55 C_{F} n_{f}^{4} T_{F}^{4}}{216}-\frac{175 C_{A} C_{F} n_{f}^{3} T_{F}^{3}}{144}-\frac{395 C_{A} n_{f}^{4} T_{F}^{4}}{1296}\right] \\
& +l_{\mu q}\left(\frac{107 C_{A}^{3}}{5184}+\frac{73}{864} C_{A}^{2} n_{f} T_{F}+\frac{131}{384} C_{A} C_{F} n_{f} T_{F}-\frac{7}{108} C_{A} n_{f}^{2} T_{F}^{2}\right. \\
& \left.\left.-\frac{1}{6} C_{F} n_{f}^{2} T_{F}^{2}+\frac{n_{f}^{3} T_{F}^{3}}{648}\right)\right\} .
\end{aligned}
$$

In [1] the three-loop logarithmic terms of (2.15) und (2.16) were constructed from the two-loop result and the requirement that $\mu^{2} \frac{d}{d \mu^{2}} C_{1, \mathrm{RGI}}^{(S, T)}$ vanishes identically. and indeed we find the same result in this explicit calculation. This requirement also explains the absence of Logarithms in the lower-order terms [1].

$$
\begin{aligned}
C_{1}^{\mathrm{GG}}=- & +a_{s}\left\{-\frac{49 C_{A}}{36}+\frac{5 n_{f} T_{F}}{9}+l_{\mu q}\left(\frac{n_{f} T_{F}}{3}-\frac{11 C_{A}}{12}\right)\right\} \\
+ & a_{s}^{2}\left\{\frac{33 C_{A}^{2} \zeta_{3}}{8}-\frac{11509 C_{A}^{2}}{1296}+\frac{3}{2} C_{A} n_{f} T_{F} \zeta_{3}+\frac{3095 C_{A} n_{f} T_{F}}{648}-3 C_{F} n_{f} T_{F} \zeta_{3}\right. \\
& +\frac{13 C_{F} n_{f} T_{F}}{4}-\frac{25 n_{f}^{2} T_{F}^{2}}{81}+l_{\mu q}\left(-\frac{1151 C_{A}^{2}}{216}+\frac{97 C_{A} n_{f} T_{F}}{27}+C_{F} n_{f} T_{F}\right. \\
& \left.-\frac{10 n_{f}^{2} T_{F}^{2}}{27}\right)+l_{\mu q}^{2}\left(-\frac{121 C_{A}^{2}}{144}+\frac{11 C_{A} n_{f} T_{F}}{18}-\frac{n_{f}^{2} T_{F}^{2}}{9}\right) \\
& \left.+\frac{1}{\varepsilon}\left[-\frac{17 C_{A}^{2}}{24}+\frac{5 C_{A} n_{f} T_{F}}{12}+\frac{C_{F} n_{f} T_{F}}{4}\right]\right\} \\
+ & a_{s}^{3}\left\{+\frac{5315 C_{A}^{3} \zeta_{3}}{144}-\frac{55 C_{A}^{3} \zeta_{5}}{8}-\frac{9775633 C_{A}^{3}}{186624}-\frac{263}{144} C_{A}^{2} n_{f} T_{F} \zeta_{3}\right. \\
& -5 C_{A}^{2} n_{f} T_{F} \zeta_{5}+\frac{1299295 C_{A}^{2} n_{f} T_{F}}{31104}-\frac{331}{16} C_{A} C_{F} n_{f} T_{F} \zeta_{3}-\frac{15}{2} C_{A} C_{F} n_{f} T_{F} \zeta_{5} \\
& +\frac{35707 C_{A} C_{F} n_{f} T_{F}}{1152}-\frac{121}{36} C_{A} n_{f}^{2} T_{F}^{2} \zeta_{3}-\frac{116773 C_{A} n_{f}^{2} T_{F}^{2}}{15552}-9 C_{F}^{2} n_{f} T_{F} \zeta_{3} \\
& +15 C_{F}^{2} n_{f} T_{F} \zeta_{5}-\frac{45}{16} C_{F}^{2} n_{f} T_{F}+\frac{13}{2} C_{F} n_{f}^{2} T_{F}^{2} \zeta_{3}-\frac{2399}{288} C_{F} n_{f}^{2} T_{F}^{2}+\frac{125 n_{f}^{3} T_{F}^{3}}{729} \\
& +l_{\mu q}\left(\frac{363 C_{A}^{3} \zeta_{3}}{32}-\frac{360325 C_{A}^{3}}{10368}+\frac{55757 C_{A}^{2} n_{f} T_{F}}{1728}-\frac{33}{4} C_{A} C_{F} n_{f} T_{F} \zeta_{3}\right. \\
& +\frac{2527}{192} C_{A} C_{F} n_{f} T_{F}-\frac{3}{2} C_{A} n_{f}^{2} T_{F}^{2} \zeta_{3}-\frac{2057}{288} C_{A} n_{f}^{2} T_{F}^{2}-\frac{9}{32} C_{F}^{2} n_{f} T_{F} \\
& \left.+3 C_{F} n_{f}^{2} T_{F}^{2} \zeta_{3}-\frac{209}{48} C_{F} n_{f}^{2} T_{F}^{2}+\frac{25 n_{f}^{3} T_{F}^{3}}{81}\right)+l_{\mu q}^{2}\left(-\frac{1793 C_{A}^{3}}{216}\right. \\
& \left.+\frac{273}{32} C_{A}^{2} n_{f} T_{F}+\frac{55}{32} C_{A} C_{F} n_{f} T_{F}-\frac{181}{72} C_{A} n_{f}^{2} T_{F}^{2}-\frac{5}{8} C_{F} n_{f}^{2} T_{F}^{2}+\frac{5 n_{f}^{3} T_{F}^{3}}{27}\right) \\
&
\end{aligned}
$$




$$
\begin{aligned}
+ & l_{\mu q}^{3}\left(-\frac{1331 C_{A}^{3}}{1728}+\frac{121}{144} C_{A}^{2} n_{f} T_{F}-\frac{11}{36} C_{A} n_{f}^{2} T_{F}^{2}+\frac{n_{f}^{3} T_{F}^{3}}{27}\right) \\
\frac{1}{\varepsilon} & {\left[+\frac{1415 C_{A}^{2} n_{f} T_{F}}{864}-\frac{2857 C_{A}^{3}}{1728}+\frac{205 C_{A} C_{F} n_{f} T_{F}}{288}\right.} \\
& \left.-\frac{79 C_{A} n_{f}^{2} T_{F}^{2}}{432}-\frac{C_{F}^{2} n_{f} T_{F}}{16}-\frac{11 C_{F} n_{f}^{2} T_{F}^{2}}{72}\right] \\
\frac{1}{\varepsilon^{2}} & {\left.\left[-\frac{89 C_{A}^{2} n_{f} T_{F}}{144}+\frac{187 C_{A}^{3}}{288}-\frac{11 C_{A} C_{F} n_{f} T_{F}}{48}+\frac{5 C_{A} n_{f}^{2} T_{F}^{2}}{36}+\frac{C_{F} n_{f}^{2} T_{F}^{2}}{12}\right]\right\} . }
\end{aligned}
$$

The tree-level, one-loop and two-loop terms in (2.17) have been computed in [9], [37, 38] and [1] correspondingly.

As already observed at two-loop level [1] there are divergent contact terms in $C_{1}^{\mathrm{GG}}$ starting from $\mathcal{O}\left(\alpha_{s}^{2}\right)$. It is intersting to observe that these divergent terms can be expressed through the $\beta$-function coefficients from (1.13):

$$
C_{1}^{\mathrm{GG}}=\frac{1}{\varepsilon}\left[-a_{s}^{2} \beta_{1}-a_{s}^{3} 2 \beta_{2}\right]+\frac{1}{\varepsilon^{2}}\left[-a_{s}^{3} \beta_{0} \beta_{1}\right]+\text { finite }
$$

This feature points to the possibility that the contact terms and hence the additive part of the renormalization of the Wilson coefficient $C_{1}^{\mathrm{GG}}$ could be expressed completely through the $\beta$-function. An explanation for this curious behaviour and its meaning for the $O_{1^{-}}$ correlator remains to be found. However, we can try to find a minimal closed formula for the representation (2.18) of the divergent part of $C_{1}^{\mathrm{GG}}$. A reasonable possibility reproducing (2.18) to the given order in $a_{s}$ is

$$
C_{1}^{\mathrm{GG}}=a_{s}^{2}\left(1-\frac{\beta\left(a_{s}\right)}{\varepsilon}\right) \frac{\partial}{\partial a_{s}}\left[\frac{\beta\left(a_{s}\right)}{\varepsilon a_{s}}\right]+\mathcal{O}\left(a_{s}^{4}\right)=\frac{a_{s}^{2}}{Z_{G}} \frac{\partial}{\partial a_{s}}\left[\frac{\beta\left(a_{s}\right)}{\varepsilon a_{s}}\right]+\mathcal{O}\left(a_{s}^{4}\right),
$$

which contains a second order derivative of $Z_{\alpha_{s}}$ w.r.t. $\alpha_{s}$. It can be hoped that an explanation for this can be found along the lines of [15], where the renormalization constant $Z_{G}$ in (1.12) was obtained by taking first order derivatives of the generating functional of QCD w.r.t. $\alpha_{s}$, the gauge parameter and the external currents. We hope to return to this question in a future publication.

An unambiguous result can be obtained for the Adler function of $C_{1}^{\mathrm{GG}}$, in which all contact terms, finite and divergent, vanish:

$$
\begin{aligned}
Q^{2} \frac{d}{d Q^{2}} C_{1}^{\mathrm{GG}}=a_{s} & \left\{\frac{11 C_{A}}{12}-\frac{n_{f} T_{F}}{3}\right\} \\
+ & a_{s}^{2}\left\{\frac{1151 C_{A}^{2}}{216}-\frac{97 C_{A} n_{f} T_{F}}{27}-C_{F} n_{f} T_{F}+\frac{10 n_{f}^{2} T_{F}^{2}}{27}\right. \\
& \left.+l_{\mu q}\left(\frac{121 C_{A}^{2}}{72}-\frac{11 C_{A} n_{f} T_{F}}{9}+\frac{2 n_{f}^{2} T_{F}^{2}}{9}\right)\right\} \\
+ & a_{s}^{3}\left\{-\frac{363 C_{A}^{3} \zeta_{3}}{32}+\frac{360325 C_{A}^{3}}{10368}-\frac{55757 C_{A}^{2} n_{f} T_{F}}{1728}\right. \\
& +\frac{33}{4} C_{A} C_{F} n_{f} T_{F} \zeta_{3}-\frac{2527}{192} C_{A} C_{F} n_{f} T_{F}+\frac{3}{2} C_{A} n_{f}^{2} T_{F}^{2} \zeta_{3}
\end{aligned}
$$




$$
\begin{aligned}
& +\frac{2057}{288} C_{A} n_{f}^{2} T_{F}^{2}+\frac{9}{32} C_{F}^{2} n_{f} T_{F}-3 C_{F} n_{f}^{2} T_{F}^{2} \zeta_{3} \\
& +\frac{209}{48} C_{F} n_{f}^{2} T_{F}^{2}-\frac{25 n_{f}^{3} T_{F}^{3}}{81}+l_{\mu q}\left(\frac{1793 C_{A}^{3}}{108}\right. \\
& -\frac{273}{16} C_{A}^{2} n_{f} T_{F}-\frac{55}{16} C_{A} C_{F} n_{f} T_{F}+\frac{181}{36} C_{A} n_{f}^{2} T_{F}^{2} \\
& \left.+\frac{5}{4} C_{F} n_{f}^{2} T_{F}^{2}-\frac{10 n_{f}^{3} T_{F}^{3}}{27}\right)+l_{\mu q}^{2}\left(\frac{1331 C_{A}^{3}}{576}\right. \\
& \left.\left.-\frac{121}{48} C_{A}^{2} n_{f} T_{F}+\frac{11}{12} C_{A} n_{f}^{2} T_{F}^{2}-\frac{n_{f}^{3} T_{F}^{3}}{9}\right)\right\}
\end{aligned}
$$

In analogy to the construction above we can also find an RGI Wilson coefficient

$$
C_{1}^{\mathrm{GG}, \mathrm{RGI}}:=\hat{\beta}\left(a_{s}\right) C_{1}^{G G},
$$

which fulfills

$$
C_{1}^{\mathrm{GG}, \mathrm{RGI}} O_{1}^{\mathrm{RGI}}=C_{1}^{\mathrm{GG}}\left[O_{1}\right] .
$$

For the derivative of the Wilson coefficient w.r.t. $Q^{2}$ we find

$$
\begin{aligned}
& Q^{2} \frac{d}{d Q^{2}} C_{1}^{\mathrm{GG}, \mathrm{RGI} I}=a_{s}^{2}\left\{\frac{11 C_{A}}{12}-\frac{n_{f} T_{F}}{3}\right\} \\
&+ a_{s}^{3}\left\{\frac{163 C_{A}^{2}}{27}-\frac{433 C_{A} n_{f} T_{F}}{108}-\frac{5 C_{F} n_{f} T_{F}}{4}+\frac{10 n_{f}^{2} T_{F}^{2}}{27}\right. \\
&\left.+l_{\mu q}\left(\frac{121 C_{A}^{2}}{72}-\frac{11 C_{A} n_{f} T_{F}}{9}+\frac{2 n_{f}^{2} T_{F}^{2}}{9}\right)\right\} \\
&+ a_{s}^{4} \frac{1}{\left(11 C_{A}-4 n_{f} T_{F}\right)}\left\{-\frac{3993 C_{A}^{4} \zeta_{3}}{32}+\frac{565933 C_{A}^{4}}{1296}+\frac{363 C_{A}^{3} n_{f} T_{F} \zeta_{3}}{8}\right. \\
&-\frac{730223 C_{A}^{3} n_{f} T_{F}}{1296}+\frac{363 C_{A}^{2} C_{F} n_{f} T_{F} \zeta_{3}}{4}-\frac{16625 C_{A}^{2} C_{F} n_{f} T_{F}}{96} \\
&+\frac{33 C_{A}^{2} n_{f}^{2} T_{F}^{2} \zeta_{3}}{2}+\frac{100667 C_{A}^{2} n_{f}^{2} T_{F}^{2}}{432}+\frac{7 C_{F}^{2} n_{f}^{2} T_{F}^{2}}{4}+\frac{55 C_{A} C_{F}^{2} n_{f} T_{F}}{16} \\
&+12 C_{F} n_{f}^{3} T_{F}^{3} \zeta_{3}-\frac{113 C_{F} n_{f}^{3} T_{F}^{3}}{6}-66 C_{A} C_{F} n_{f}^{2} T_{F}^{2} \zeta_{3}+\frac{1423 C_{A} C_{F} n_{f}^{2} T_{F}^{2}}{12} \\
&+\frac{100 n_{f}^{4} T_{F}^{4}}{81}-6 C_{A} n_{f}^{3} T_{F}^{3} \zeta_{3}-\frac{11075 C_{A} n_{f}^{3} T_{F}^{3}}{324} \\
&+l_{\mu q}\left(\frac{85063 C_{A}^{4}}{432}-\frac{117887 C_{A}^{3} n_{f} T_{F}}{432}-\frac{2057 C_{A}^{2} C_{F} n_{f} T_{F}}{48}+\frac{1184 C_{A}^{2} n_{f}^{2} T_{F}^{2}}{9}\right. \\
&\left.-\frac{17 C_{F} n_{f}^{3} T_{F}^{3}}{3}+\frac{187 C_{A} C_{F} n_{f}^{2} T_{F}^{2}}{6}+\frac{40 n_{f}^{4} T_{F}^{4}}{27}-\frac{683 C_{A} n_{f}^{3} T_{F}^{3}}{27}\right) \\
&+l_{\mu q}^{2}\left(\frac{14641 C_{A}^{4}}{576}-\frac{1331 C_{A}^{3} n_{f} T_{F}}{36}+\frac{121 C_{A}^{2} n_{f}^{2} T_{F}^{2}}{6}\right. \\
&\left.\left.+\frac{4 n_{f}^{4} T_{F}^{4}}{9}-\frac{44 C_{A} n_{f}^{3} T_{F}^{3}}{9}\right)\right\} \cdot \\
& 9
\end{aligned}
$$




\section{$3 \quad$ Numerics}

Finally, we consider two cases which are interesting for applications numerically, that is gluodynamics $\left(n_{f}=0\right)$ and QCD with only three light quarks $\left(n_{f}=3\right)$. For this we choose the scale $\mu^{2}=Q^{2}$, i.e. we set $l_{\mu q}=0$. For the correlator (1.15) we find

$$
\begin{aligned}
& C_{1}^{(S)}\left(\mu^{2}=Q^{2}, n_{f}=0\right)=\frac{22}{9} a_{s}\left(1+0.943182 a_{s}-7.06061 a_{s}^{2}\right), \\
& C_{1}^{(S)}\left(\mu^{2}=Q^{2}, n_{f}=3\right)=2 a_{s}\left(1+0.652778 a_{s}-5.18519 a_{s}^{2}\right), \\
& C_{1}^{(T)}\left(\mu^{2}=Q^{2}, n_{f}=0\right)=-\frac{5}{6} a_{s}\left(1+2.075 a_{s}+14.3904 a_{s}^{2}\right), \\
& C_{1}^{(T)}\left(\mu^{2}=Q^{2}, n_{f}=3\right)=-\frac{15}{16} a_{s}\left(1+0.444444 a_{s}+6.64113 a_{s}^{2}\right) .
\end{aligned}
$$

and for (1.16) we get

$$
\begin{aligned}
& Q^{2} \frac{d}{d Q^{2}} C_{1}^{\mathrm{GG}}\left(\mu^{2}=Q^{2}, n_{f}=0\right)=\frac{11}{4} a_{s}^{2}\left(1+17.4394 a_{s}+207.338 a_{s}^{2}\right), \\
& Q^{2} \frac{d}{d Q^{2}} C_{1}^{\mathrm{GG}}\left(\mu^{2}=Q^{2}, n_{f}=3\right)=\frac{9}{4} a_{s}^{2}\left(1+13.6111 a_{s}+78.8642 a_{s}^{2}\right) .
\end{aligned}
$$

For the RGI coefficients the numerical evaluation yields

$$
\begin{aligned}
& C_{1, \mathrm{RGI}}^{(S)}\left(\mu^{2}=Q^{2}, n_{f}=0\right)=\frac{22}{9}\left(1-1.375 a_{s}-11.9896 a_{s}^{2}\right), \\
& C_{1, \mathrm{RGI}}^{(S)}\left(\mu^{2}=Q^{2}, n_{f}=3\right)=2\left(1-1.125 a_{s}-7.65625 a_{s}^{2}\right), \\
& C_{1, \mathrm{RGI}}^{(T)}\left(\mu^{2}=Q^{2}, n_{f}=0\right)=-\frac{5}{6}\left(1-0.2431825 a_{s}+6.83767 a_{s}^{2}\right), \\
& C_{1, \mathrm{RGI}}^{(T)}\left(\mu^{2}=Q^{2}, n_{f}=3\right)=-\frac{15}{16}\left(1-1.33333 a_{s}+4.54043 a_{s}^{2}\right)
\end{aligned}
$$

and

$$
\begin{aligned}
& Q^{2} \frac{d}{d Q^{2}} C_{1}^{\mathrm{GG}, \mathrm{RGI}}\left(\mu^{2}=Q^{2}, n_{f}=0\right)=\frac{11}{4} a_{s}\left(1+19.7576 a_{s}+255.882 a_{s}^{2}\right), \\
& Q^{2} \frac{d}{d Q^{2}} C_{1}^{\mathrm{GG}, \mathrm{RGI}}\left(\mu^{2}=Q^{2}, n_{f}=3\right)=\frac{9}{4} a_{s}\left(1+15.3889 a_{s}+107.533 a_{s}^{2}\right) .
\end{aligned}
$$

The numerical impact of the higher order corrections can be seen by evaluating the RGI coefficients at $\mu=M_{Z}, \mu=3.5 \mathrm{GeV}$ and $\mu=2 \mathrm{GeV}$, where

$$
\alpha_{s}^{\left(n_{f}=5\right)}\left(M_{Z}\right) \approx 0.118, \alpha_{s}^{\left(n_{f}=3\right)}(3.5 \mathrm{GeV}) \approx 0.24 \text { and } \alpha_{s}^{\left(n_{f}=3\right)}(2 \mathrm{GeV}) \approx 0.30[39]
$$

for the cases $n_{f}=5$ and $n_{f}=3$ respectively. We find

$$
C_{1, \mathrm{RGI}}^{(S)}\left(Q^{2}=\mu^{2}=M_{Z}^{2}, n_{f}=5\right)=\frac{46}{27}(-\underbrace{0.00705235}_{3 \text { loop }}-\underbrace{0.0359955}_{2 \text { loop }}+\underbrace{1}_{1 \text { loop }}),
$$




$$
\begin{gathered}
C_{1, \mathrm{RGI}}^{(S)}\left(Q^{2}=\mu^{2}=(3.5 \mathrm{GeV})^{2}, n_{f}=3\right)=2(-\underbrace{0.0446826}_{3 \text { loop }}-\underbrace{0.0859437}_{2 \text { loop }}+\underbrace{1}_{1 \text { loop }}), \\
C_{1, \mathrm{RGI}}^{(S)}\left(Q^{2}=\mu^{2}=(2 \mathrm{GeV})^{2}, n_{f}=3\right)=2(-\underbrace{0.0698166}_{3 \text { loop }}-\underbrace{0.10743}_{2 \text { loop }}+\underbrace{1}_{1 \text { loop }})
\end{gathered}
$$

and

$$
\begin{gathered}
C_{1, \mathrm{RGI}}^{(T)}\left(Q^{2}=\mu^{2}=M_{Z}^{2}, n_{f}=5\right)=-\frac{145}{144}(\underbrace{0.00930401}_{3 \text { loop }}-\underbrace{0.0640238}_{2 \text { loop }}+\underbrace{1}_{1 \text { loop }}), \\
C_{1, \mathrm{RGI}}^{(T)}\left(Q^{2}=\mu^{2}=(3.5 \mathrm{GeV})^{2}, n_{f}=3\right)=-\frac{15}{16}(\underbrace{0.0264984}_{3 \text { loop }}-\underbrace{0.101859}_{2 \text { loop }}+\underbrace{1}_{1 \text { loop }}), \\
C_{1, \mathrm{RGI}}^{(T)}\left(Q^{2}=\mu^{2}=(2 \mathrm{GeV})^{2}, n_{f}=3\right)=-\frac{15}{16}(\underbrace{0.0414038}_{3 \text { loop }}-\underbrace{0.127324}_{2 \text { loop }}+\underbrace{1}_{1 \text { loop }}) .
\end{gathered}
$$

for the correlator (1.15). This shows that for the energy-momentum tensor the Wilson coefficient $C_{1}^{(T)}$ is well convergent, even at $\mu=2 \mathrm{GeV}$. The three-loop approximation for $C_{1}^{(S)}$ at low scales is less good, but still acceptable. At $\mu=3.5 \mathrm{GeV}$ the three-loop correction is $50 \%$ of the two-loop correction but both together are only a $12 \%$ correction to the one-loop result.

For the correlator (1.16) we find with

$$
\alpha_{s}^{\left(n_{f}=3\right)}(5 \mathrm{GeV}) \approx 0.213[39]
$$

in addition to (3.13):

$$
\begin{aligned}
& Q^{2} \frac{d}{d Q^{2}} C_{1}^{\mathrm{GG}, \mathrm{RGI}}\left(Q^{2}=\mu^{2}=M_{Z}^{2}, n_{f}=5\right) \\
& =\frac{23}{12} a_{s}^{2}\left(\mu=M_{Z}\right)(\underbrace{0.0074766}_{3 \text { loop }}+\underbrace{0.439205}_{2 \text { loop }}+\underbrace{1}_{1 \text { loop }}) \text {, } \\
& Q^{2} \frac{d}{d Q^{2}} C_{1}^{\mathrm{GG}, \mathrm{RGI}}\left(Q^{2}=\mu^{2}=(5 \mathrm{GeV})^{2}, n_{f}=3\right) \\
& =\frac{9}{4} a_{s}^{2}(\mu=5 \mathrm{GeV})(\underbrace{0.494311}_{3 \text { loop }}+\underbrace{1.04337}_{2 \text { loop }}+\underbrace{1}_{1 \text { loop }}) \text {, } \\
& Q^{2} \frac{d}{d Q^{2}} C_{1}^{\mathrm{GG}, \mathrm{RGI}}\left(Q^{2}=\mu^{2}=(2 \mathrm{GeV})^{2}, n_{f}=3\right) \\
& =\frac{9}{4} a_{s}^{2}(\mu=2 \mathrm{GeV})(\underbrace{0.980582}_{3 \text { loop }}+\underbrace{1.46953}_{2 \text { loop }}+\underbrace{1}_{1 \text { loop }}) \text {. }
\end{aligned}
$$


Here the convergence at low scales is not so good as the two-loop correction becomes larger than the one-loop correction at $\mu=5 \mathrm{GeV}$ and the three-loop correction shifts the result by another $50 \%$ of the one-loop results. This suggests that higher order corrections should always be taken into account when this coefficient is used e.g. in sum rules and special care has to be taken with regard to the convergence of the perturbation series at the scale where perturbative and non-perturbative physics are separated in the OPE. With this in mind, extending $C_{1}^{\mathrm{GG}}$ to even higher orders in the future could therefore be an interesting task.

\section{Conclusions}

We have presented the missing three-loop corrections to the OPE of the correlator of two scalar gluonic operators $\left[O_{1}\right]=-\frac{Z_{G}}{4} G^{\mathrm{B} a \mu \nu} G_{\mu \nu}^{\mathrm{B} a}$ and of the correlator of two energymomentum tensors $T^{\mu \nu}$ in massless QCD at zero temperature.

These are the three-loop contributions to the coefficient $C_{1}$ in front of the local operator $\left[O_{1}\right]$. We have also constructed renormalization group invariant versions of these coefficients and confirmed the predictions made in [1] for the logarithmic part of these coefficients.

In the coefficient $C_{1}^{\mathrm{GG}}$ for the $O_{1}$-correlator we observe the curious feature that divergent contact terms appear which are expressible through the QCD $\beta$-function. These constact terms as well as contact terms in the $T^{\mu \nu}$-correlator proportional to the tensor structures $t_{4}^{\mu \nu ; \rho \sigma}(q)$ and $t_{5}^{\mu \nu ; \rho \sigma}(q)$ from (2.1) have to be subtracted. If we consider only derivatives w.r.t. $Q^{2}$ of ambiguous Wilson coefficients these terms vanish automatically.

All results can be found in a machine-readable form at http://www-ttp.particle.uni-karlsruhe.de/Progdata/ttp14/ttp14-023/.

\section{Acknowledgments}

I thank K. G. Chetyrkin for many useful discussions and his collaboration on the previous project [1]. I am also grateful to J. H. Kühn for his support and useful comments. This work has been supported by the Deutsche Forschungsgemeinschaft in the Sonderforschungsbereich/Transregio SFB/TR-9 "Computational Particle Physics", the Graduiertenkolleg "Elementarteilchenphysik bei höchsten Energien und höchster Präzission" and the "Karlsruhe School of Elementary Particle and Astroparticle Physics: Science and Technology (KSETA)".

Open Access. This article is distributed under the terms of the Creative Commons Attribution License (CC-BY 4.0), which permits any use, distribution and reproduction in any medium, provided the original author(s) and source are credited.

\section{References}

[1] M.F. Zoller and K.G. Chetyrkin, OPE of the energy-momentum tensor correlator in massless QCD, JHEP 12 (2012) 119 [arXiv:1209.1516] [INSPIRE]. 
[2] P.A. Baikov and K.G. Chetyrkin, Top Quark Mediated Higgs Boson Decay into Hadrons to Order $\alpha_{s}^{5}$, Phys. Rev. Lett. 97 (2006) 061803 [hep-ph/0604194] [InSPIRE].

[3] M.F. Zoller, OPE of the pseudoscalar gluonium correlator in massless QCD to three-loop order, JHEP 07 (2013) 040 [arXiv: 1304.2232] [INSPIRE].

[4] H. Forkel, Direct instantons, topological charge screening and QCD glueball sum rules, Phys. Rev. D 71 (2005) 054008 [hep-ph/0312049] [INSPIRE].

[5] K.G. Wilson, Nonlagrangian models of current algebra, Phys. Rev. 179 (1969) 1499 [INSPIRE].

[6] S.G. Gorishnii, S.A. Larin and F.V. Tkachov, The algorithm for ope coefficient functions in the MS scheme, Phys. Lett. B 124 (1983) 217 [inSPIRE].

[7] S.G. Gorishnii and S.A. Larin, Coefficient Functions of Asymptotic Operator Expansions in Minimal Subtraction Scheme, Nucl. Phys. B 283 (1987) 452 [InSPIRE].

[8] M.A. Shifman, A.I. Vainshtein and V.I. Zakharov, QCD and Resonance Physics. Sum Rules, Nucl. Phys. B 147 (1979) 385 [InSPIRE].

[9] V.A. Novikov, M.A. Shifman, A.I. Vainshtein and V.I. Zakharov, In a Search for Scalar Gluonium, Nucl. Phys. B 165 (1980) 67 [INSPIRE].

[10] V.A. Novikov, M.A. Shifman, A.I. Vainshtein and V.I. Zakharov, $\eta$-prime Meson as Pseudoscalar Gluonium, Phys. Lett. B 86 (1979) 347 [INSPIRE].

[11] M. Bochicchio and S.P. Muscinelli, Ultraviolet asymptotics of glueball propagators, JHEP 08 (2013) 064 [arXiv: 1304.6409] [InSPIRE].

[12] M. Bochicchio, Yang-Mills mass gap, Floer homology, glueball spectrum and conformal window in large- $N$ QCD, arXiv:1312.1350 [INSPIRE].

[13] N.K. Nielsen, The Energy Momentum Tensor in a Nonabelian Quark Gluon Theory, Nucl. Phys. B 120 (1977) 212 [inSPIRE].

[14] N.K. Nielsen, Gauge Invariance and Broken Conformal Symmetry, Nucl. Phys. B 97 (1975) 527 [inSPIRE].

[15] V. Spiridonov, Anomalous Dimension of $G_{\mu \nu}^{2}$ and $\beta$-function, Preprint IYAI-P-0378 (1984).

[16] T. van Ritbergen, J.A.M. Vermaseren and S.A. Larin, The Four loop $\beta$-function in quantum chromodynamics, Phys. Lett. B 400 (1997) 379 [hep-ph/9701390] [INSPIRE].

[17] M. Czakon, The Four-loop QCD $\beta$-function and anomalous dimensions, Nucl. Phys. B 710 (2005) 485 [hep-ph/0411261] [INSPIRE].

[18] S.A. Larin, The Renormalization of the axial anomaly in dimensional regularization, Phys. Lett. B 303 (1993) 113 [hep-ph/9302240] [INSPIRE].

[19] A.L. Kataev, N.V. Krasnikov and A.A. Pivovarov, Two Loop Calculations for the Propagators of Gluonic Currents, Nucl. Phys. B 198 (1982) 508 [Erratum ibid. B 490 (1997) 505] [hep-ph/9612326] [INSPIRE].

[20] K.G. Chetyrkin, B.A. Kniehl and M. Steinhauser, Hadronic Higgs decay to order alpha- $S^{4}$, Phys. Rev. Lett. 79 (1997) 353 [hep-ph/9705240] [INSPIRE].

[21] H.B. Meyer, Energy-momentum tensor correlators and viscosity, PoS (LATTICE 2008) 017 [arXiv:0809.5202] [INSPIRE]. 
[22] H.B. Meyer, A Calculation of the shear viscosity in $\mathrm{SU}(3)$ gluodynamics, Phys. Rev. D 76 (2007) 101701 [arXiv:0704.1801] [INSPIRE].

[23] H.B. Meyer, Energy-momentum tensor correlators and spectral functions, JHEP 08 (2008) 031 [arXiv:0806.3914] [INSPIRE].

[24] J.C. Collins, A. Duncan and S.D. Joglekar, Trace and Dilatation Anomalies in Gauge Theories, Phys. Rev. D 16 (1977) 438 [InSPIRE].

[25] M. Laine, Thermal 2-loop master spectral function at finite momentum, JHEP 05 (2013) 083 [arXiv: 1304.0202] [INSPIRE].

[26] K. Kajantie, M. Krssak and A. Vuorinen, Energy momentum tensor correlators in hot Yang-Mills theory: holography confronts lattice and perturbation theory, JHEP 05 (2013) 140 [arXiv: 1302.1432] [INSPIRE].

[27] Y. Zhu and A. Vuorinen, The shear channel spectral function in hot Yang-Mills theory, JHEP 03 (2013) 002 [arXiv:1212.3818] [INSPIRE].

[28] M. Laine, M. Vepsäläinen and A. Vuorinen, Ultraviolet asymptotics of scalar and pseudoscalar correlators in hot Yang-Mills theory, JHEP 10 (2010) 010 [arXiv:1008.3263] [INSPIRE].

[29] Y. Schröder, M. Vepsäläinen, A. Vuorinen and Y. Zhu, The Ultraviolet limit and sum rule for the shear correlator in hot Yang-Mills theory, JHEP 12 (2011) 035 [arXiv:1109.6548] [INSPIRE].

[30] A.A. Pivovarov, Two loop corrections to the correlator of tensor currents in gluodynamics, Phys. Atom. Nucl. 63 (2000) 1646 [hep-ph/9905485] [INSPIRE].

[31] J.A.M. Vermaseren, Axodraw, Comput. Phys. Commun. 83 (1994) 45 [InSPIRE].

[32] P. Nogueira, Automatic Feynman graph generation, J. Comput. Phys. 105 (1993) 279 [INSPIRE].

[33] J.A.M. Vermaseren, New features of FORM, math-ph/0010025 [INSPIRE].

[34] M. Tentyukov and J.A.M. Vermaseren, The Multithreaded version of FORM, Comput. Phys. Commun. 181 (2010) 1419 [hep-ph/0702279] [INSPIRE].

[35] S.G. Gorishnii, S.A. Larin, L.R. Surguladze and F.V. Tkachov, Mincer: Program for Multiloop Calculations in Quantum Field Theory for the Schoonschip System, Comput. Phys. Commun. 55 (1989) 381 [INSPIRE].

[36] T. van Ritbergen, A.N. Schellekens and J.A.M. Vermaseren, Group theory factors for Feynman diagrams, Int. J. Mod. Phys. A 14 (1999) 41 [hep-ph/9802376] [INSPIRE].

[37] E. Bagan and T.G. Steele, Infrared Aspects of the One Loop, Scalar Glueball Operator Product Expansion, Phys. Lett. B 234 (1990) 135 [INSPIRE].

[38] D. Harnett and T.G. Steele, Quark effects in the gluon condensate contribution to the scalar glueball correlation function, JHEP 12 (2004) 037 [hep-ph/0410388] [INSPIRE].

[39] K.G. Chetyrkin, J.H. Kuhn and M. Steinhauser, RunDec: A Mathematica package for running and decoupling of the strong coupling and quark masses, Comput. Phys. Commun. 133 (2000) 43 [hep-ph/0004189] [INSPIRE]. 